\title{
MRI Evidence of Impaired CSF Homeostasis in Obesity- Associated Idiopathic Intracranial Hypertension
}

\author{
N. Alperin, S. Ranganathan, A.M. Bagci, D.J. Adams, B. Ertl-Wagner, E. Saraf-Lavi, E.M. Sklar, and B.L. Lam
}

O- EBM

\begin{abstract}
BACKGROUND AND PURPOSE: Impaired CSF homeostasis and altered venous hemodynamics are proposed mechanisms for elevated pressure in IIH. However, the lack of ventricular expansion steered the focus away from CSF homeostasis in IIH. This study aims to measure intracranial CSF volumes and cerebral venous drainage with MR imaging to determine whether increased CSF volume from impaired CSF homeostasis and venous hemodynamics occur in obesity-related IIH.
\end{abstract}

MATERIALS AND METHODS: Two homogeneous cohorts of 11 newly diagnosed pretreatment overweight women with IIH and 11 overweight healthy women were prospectively studied. 3D volumetric MR imaging of the brain was used to quantify CSF and brain tissue volumes, and dynamic phase contrast was used to measure relative cerebral drainage through the internal jugular veins.

RESULTS: Findings confirm normal ventricular volume in IIH. However, extraventricular CSF volume is significantly increased in IIH (290 \pm 52 versus $220 \pm 24 \mathrm{~mL}, P=.001)$. This is even more significant after normalization with intracranial volume $(P=.0007)$. GM interstitial fluid volume is also increased in $\mathrm{IIH}(602 \pm 57$ versus $557 \pm 31 \mathrm{~mL}, P=.037)$. Total arterial inflow is normal, but relative venous drainage through the IJV is significantly reduced in $\mathrm{IIH}(65 \pm 7 \%$ versus $81 \pm 10 \%, P=.001)$.

CONCLUSIONS: Increased intracranial CSF volume that accumulates in the extraventricular subarachnoid space provides direct evidence for impaired CSF homeostasis in obesity-associated IIH. The finding of larger GM interstitial fluid volume is consistent with increased overall resistance to cerebral venous drainage, as evident from reduced relative cerebral drainage through the IJV. The present study confirms that both impaired CSF homeostasis and venous hemodynamics coexist in obesity-associated IIH.

ABBREVIATIONS: $\mathrm{GM}=$ gray matter; $\mathrm{ICV}=$ intracranial volume; $\mathrm{IIH}=$ idiopathic intracranial hypertension; IJV = internal jugular vein

diopathic intracranial hypertension, or pseudotumor cerebri, is a debilitating neurologic disorder characterized by elevated intracranial pressure of unknown cause. ${ }^{1}$ IIH is manifested mainly by severe headaches and often with visual impairments caused by increased CSF pressure on the optic nerve and globe. ${ }^{1}$ IIH can occur both in males and females of a wide age range, though it is

Received January 29, 2012; accepted after revision March 30.

From the Department of Radiology (N.A., S.R., A.M.B., E.S.-L., E.M.S.), Bascom Palmer Eye Institute, Department of Ophthalmology (B.L.L.), and Department of Neurology (D.J.A.) University of Miami, Miami, Florida; and Department of Radiology (B.E.-W.), University of Munich, Munich, Germany.

This research was supported in part by the National Institutes of Health (R01NS052122).

Please address correspondence to Noam Alperin, PhD, Department of Radiology, Professional Arts Center, University of Miami Leonard M. Miller School of Medicine, 1150 NW 14th St, Suite713 (M-869), Miami, FL 33136; e-mail: Nalperin@med.miami.edu

- Indicates open access to non-subscribers at www.ajnr.org

Evidence-Based Medicine Level 2.

http://dx.doi.org/10.3174/ajnr.A3171 most frequent in overweight women of childbearing age, with an incidence of approximately 23 per $100,000 .^{2}$

While the precise etiology leading to the elevated intracranial pressure in IIH is largely unknown, a recent comprehensive review lists 5 possible mechanisms that can be classified into those related to disturbance of CSF homeostasis and those related to impaired venous hemodynamics. ${ }^{3}$ Impaired CSF homeostasis is assumed to occur either due to reduced CSF absorption and/or increased CSF formation. ${ }^{4}$ Mechanisms related to impaired venous hemodynamics are of either intrinsic (eg, stenosis) and/or extrinsic origin (eg, compressed sinuses, increased venous pressure) ${ }^{5,6}$ While these etiologies are listed as separate mechanisms, it is plausible that the 2 types are coupled, as increased venous pressure reduces CSF absorption. ${ }^{7}$ Critics of impaired CSF homeostasis point to the lack of ventricular expansion in IIH that occurs in other etiologies associated with impaired CSF homeostasis. ${ }^{8}$

Surprisingly, measurements of intracranial CSF volumes in $\mathrm{IIH}$ are scarce, especially of the extraventricular CSF. An early MR imaging study suggested that both subarachnoid and ventricular 
spaces are larger in IIH but argued that a wide variability limits clinical utilization for these measurements. ${ }^{9}$ In addition, limited and inconsistent reports of normative values of extraventricular CSF volumes ${ }^{10,11}$ confound the question of whether total intracranial CSF volume is increased in IIH.

The present study aims to measure intracranial CSF volumes and cerebral venous drainage with $\mathrm{MR}$ imaging to determine whether increased CSF volume from impaired CSF homeostasis and altered venous hemodynamics occur in obesity-related IIH. Extra- and intraventricular CSF volumes are measured using semiautomated segmentation of 3D volumetric T1-weighted imaging, and venous hemodynamics are assessed using phase-contrast and volumetric flow measurements of total arterial inflow and venous outflow through the jugular veins.

\section{MATERIALS AND METHODS \\ Study Participants}

A homogeneous cohort of overweight women of childbearing age with a confirmed diagnosis of IIH, as per the modified Dandy criteria, ${ }^{12}$ and an age- and weight-matched cohort of control subjects were prospectively enrolled. All study participants provided written informed consent, approved by the institutional review board, before participation in the study. A total of 11 newly diagnosed untreated patients with IIH (mean age $29 \pm 9$ years, age range $17-44$ years; mean body mass index $34.2 \pm 5.1 \mathrm{~kg} / \mathrm{m}^{2}$, range $23.9-41.2 \mathrm{~kg} / \mathrm{m}^{2}$ ) and 11 healthy women (mean age $31 \pm 9$ years, age range $22-50$ years; mean body mass index $36.6 \pm 4.9 \mathrm{~kg} / \mathrm{m}^{2}$, range $30.9-48.4 \mathrm{~kg} / \mathrm{m}^{2}$ ) were studied. Detailed neurologic symptoms and ophthalmologic assessments were obtained before the MR imaging scan and diagnostic lumbar puncture. Nine of 11 patients with IIH reported moderate to severe headaches; 8 patients reported vision-related problems, including blurriness, double vision, transient visual obscurations, and impaired peripheral vision. Bilateral papilledema (range grade 1 to 4 ) was found in all patients with IIH. The mean opening pressure recorded during the diagnostic lumbar puncture was $37.23 \pm 6.87 \mathrm{~cm} \mathrm{H}_{2} \mathrm{O}$ (range $26-47 \mathrm{~cm} \mathrm{H}_{2} \mathrm{O}$ ). Control subjects were obese but otherwise healthy, without headache, visual symptoms, or papilledema.

\section{MR Imaging Scan Protocol and Data Analysis}

CSF and Brain Tissue Volumetric Assessment. MR imaging studies were performed using 1.5 and 3T scanners (Symphony and Trio; Siemens, Erlangen, Germany), both equipped with the total image matrix radio-frequency architecture. Scans were performed before diagnostic lumbar puncture and initiation of treatment. Volumetric assessment of the various cranial compartments was obtained from a 3D T1-weighted sequence (magnetization-prepared rapid acquisition of gradient echo) with an isotropic resolution of $1 \mathrm{~mm}$. The following scan parameters were used: TR of $1700 \mathrm{~ms}$ (1900 ms for 3T), TE $3.53 \mathrm{~ms}$ (4.6 for 3T), TI $1100 \mathrm{~ms}$, flip angle 15 , and bandwidth of $130 \mathrm{~Hz} /$ pixel. Potential for bias due to field strength was assessed by repeated scans in 2 test subjects.

Segmentation of the intracranial space into GM, WM, and CSF was completed by a single observer using the FMRIB Software Library package ${ }^{13,14}$ (FMRIB Software Library, Oxford, United Kingdom; http://www.fmrib.ox.ac.uk/fsl). The brain ex- traction tool ${ }^{15}$ was used to automatically identify and separate the cranium from the skull and generate a brain mask. Manual editing by a graduate student with 2 years of experience (S.R.) was used to exclude bone and eye regions, as well as modify inner skull boundaries incorrectly delineated by the brain extraction tool. Segmentation of the edited volume into GM, WM, and CSF was achieved by using the $\mathrm{FAST}^{16}$ tool, based on image intensity distribution. The total intracranial volume was then calculated as the sum of the GM, WM, and CSF volumes. Ventricular CSF volume was quantified by using the 3D Slicer software ${ }^{17}$ (version 3.6, http:// www.slicer.org). The ventricular regions, including the lateral, third, and fourth ventricles, and the cerebral aqueduct were segmented on the same T1-weighted volume. Initially, an automated region growing algorithm was used to extract the ventricles using a predetermined threshold. The resulting volume was then manually edited by the same graduate student to correctly delineate the ventricular boundaries. The extraventricular CSF volume was then computed as the difference between the intracranial CSF volume and the ventricular volume. Volume measurements were also normalized to the total intracranial volume.

Assessment of Venous Drainage through the Internal Jugular Veins. Venous drainage through the internal jugular veins in the supine position was obtained by summation of the volumetric flow rates through the right and left internal jugular veins. Total arterial inflow was obtained by summation of the volumetric flows through the internal carotid and vertebral arteries. As in steady state, total venous outflow equals total arterial inflow, the normalized jugular drainage is the volumetric flow rate through the jugular veins divided by the total arterial inflow. A dynamic phase-contrast technique with velocity encoding of 70-90 cm/s was used to measure total cerebral arterial inflow and venous outflow through the internal jugular veins. A retrospectively gated cine phase-contrast scan with the following scanning parameters was used: TR of 45-51 ms, TE of 7.4 to $9.9 \mathrm{~ms}$, flip angle of $20^{\circ}$, FOV of $14 \times 12.7 \mathrm{~cm}$, acquisition matrix of $256 \times 162$, and section thickness of $6 \mathrm{~mm}$, with 1 average. Volumetric flow rate through each IJV was obtained using the pulsatility based segmentation method for automated quantitation of volumetric flow rates through lumens conducting nonsteady flow. ${ }^{18}$ It has been shown that the pulsatility-based segmentation method improves measurement accuracy and reproducibility by using the entire time-series at each pixel location to differentiate between lumen and background regions. ${ }^{18}$

Statistical Analysis. A 2-tailed unpaired Student $t$ test was applied to test for significance of differences between volume measurements obtained from the 2 cohorts. The Pearson correlation coefficient was calculated to test for possible association between volumetric measures and severity of headache and papilledema grade. A 1-way analysis of covariance was applied to test for possible biases in the volumetric measures due to the 2 different field strengths used for scanning. The Levene test for equality of variances was performed to assess the data homogeneity required for the analysis of covariance test. Receiver operating characteristic analysis was performed to determine area under the curve and optimal sensitivity and specificity of the normalized extraventric- 

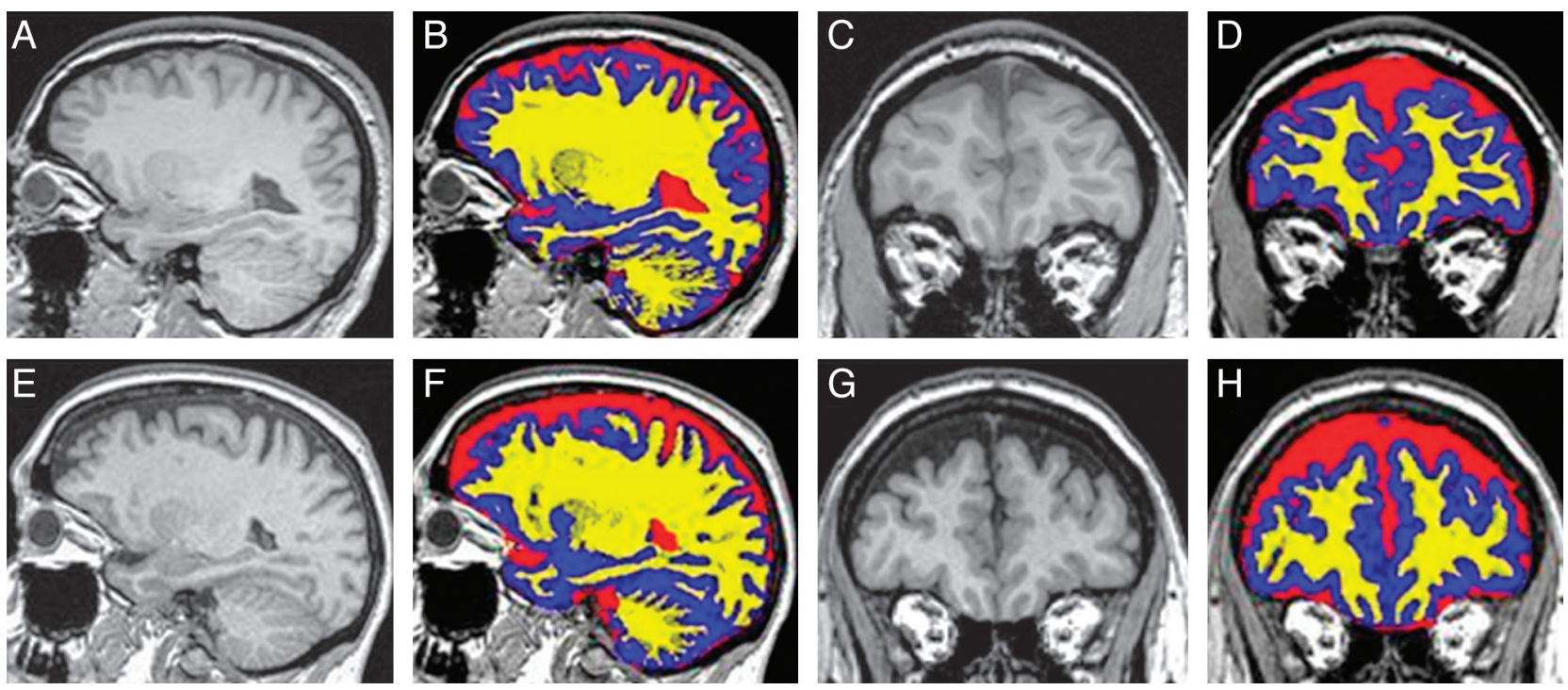

FIG 1. Segmentation of T1-weighted MR images into GM, WM, and CSF regions. Examples of T1-weighted MR images in sagittal (A, E) and coronal $(C, G)$ planes are shown without and with color overlay of the tissue segmentation into GM (blue), WM (yellow), and CSF (red) from a control subject (top row) and a patient with IIH (bottom row). Relatively larger subarachnoid CSF spaces can be visualized in the floor of the anterior cranial fossa and in the extra-axial spaces overlying the convexities in IIH.

Table 1: Mean and SD values of cranial volumetric measurements

\begin{tabular}{lccccccccc}
\hline & $\begin{array}{c}\text { ICCSF } \\
(\mathbf{m L})\end{array}$ & $\begin{array}{c}\text { VCSF } \\
(\mathbf{m L})\end{array}$ & $\begin{array}{c}\text { EVCSF } \\
(\mathbf{m L})\end{array}$ & $\begin{array}{c}\text { GM } \\
(\mathbf{m L})\end{array}$ & $\begin{array}{c}\text { WM } \\
(\mathbf{m L})\end{array}$ & $\begin{array}{c}\text { ICV } \\
(\mathbf{m L})\end{array}$ & $\begin{array}{c}\text { Nor ICCSF } \\
(\%)\end{array}$ & $\begin{array}{c}\text { Nor VCSF } \\
(\%)\end{array}$ & $\begin{array}{c}\text { Nor EVCSF } \\
(\%)\end{array}$ \\
\hline Control $(n=11)$ & $238 \pm 25$ & $19 \pm 6$ & $220 \pm 24$ & $557 \pm 31$ & $537 \pm 47$ & $1332 \pm 81$ & $18 \pm 2$ & $1.4 \pm 0.5$ & $16 \pm 1$ \\
IIH $(n=11)$ & $309 \pm 56$ & $19 \pm 7$ & $290 \pm 52$ & $602 \pm 57$ & $528 \pm 35$ & $1438 \pm 124$ & $21 \pm 3$ & $1.3 \pm 0.4$ & $20 \pm 2$ \\
$P$ value & 0.002 & 0.950 & 0.0011 & 0.038 & 0.616 & 0.029 & 0.0014 & 0.511 & 0.0007 \\
\hline
\end{tabular}

Note:-EVCSF indicates extraventricular CSF volume; ICCSF, intracranial CSF volume; Nor, normalized; VCSF, ventricular CSF volume.

ular CSF volume. Statistical analyses were performed using statistical software (MedCalc v12.0.4, Mariakerke, Belgium).

\section{RESULTS}

\section{CSF and Brain Tissue Volumes}

Analysis of covariance confirmed that there was no bias or significant differences in any of the volumetric measures due to scanner field strength. An example of sagittal and coronal T1-weighted images without and with the color overlay of the tissue segmentation from representative control and IIH subjects is shown in Fig 1. Relatively larger subarachnoid CSF spaces can be visualized both in the floor of the anterior cranial fossa and in the extra-axial spaces overlying the convexities in the IIH subjects. Mean and SD values of volumetric intracranial GM, WM, and CSF compartments are summarized in Table 1. While ventricular CSF volume is similar in both cohorts (IIH versus controls: $19 \pm 6$ versus $19 \pm$ $7 \mathrm{~mL}, P=.95$ ), a significantly larger mean intracranial CSF volume is measured in the IIH cohort compared with the healthy cohort $(309 \pm 56$ versus $238 \pm 25 \mathrm{~mL}, P=.002)$. This implies that the increased intracranial CSF volume accumulates in the extraventricular spaces (IIH versus controls: $290 \pm 52$ versus $220 \pm$ $24 \mathrm{~mL}, P=.0011)$. Differences in extraventricular CSF volume normalized for the total intracranial volume are even more statistically significant, with a $P$ value of .0007 , as these account for the intersubject variability in ICV. Sensitivity and specificity of the normalized extraventricular CSF volume measurements determined by the receiver operating characteristic analysis were $73 \%$ and $100 \%$, respectively, with an area under the curve of 0.91 .
Other significant differences were a larger GM volume in patients with IIH compared with the controls, $602 \pm 57$ versus $557 \pm 31$ $\mathrm{mL}(P=.037)$, respectively, and a larger total intracranial volume, $1438 \pm 124$ versus $1332 \pm 81 \mathrm{~mL}(P=.029)$, respectively. The volume of the WM, on the other hand, was similar in the 2 cohorts, $528 \pm 35$ versus $537 \pm 47 \mathrm{~mL}(P=.62)$. Correlations of the volumetric measures with measures of severity of headaches or papilledema grade were not statistically significant.

\section{Venous Drainage through the Internal Jugular Veins}

Mean and SD values of total CBF, volumetric flow rates through the right and left internal jugular veins, and absolute and relative drainage through the jugular veins for the 2 cohorts are summarized in Table 2. Examples of an MRV image of the cervical veins, a velocity-encoded phase-contrast image, and derived arterial inflow and venous outflow through the jugular veins in representative control and IIH subjects are shown in Fig 2. Mean total arterial inflows in the IIH and control cohorts of $823 \pm 68$ and $789 \pm$

Table 2: Mean and SD values of arterial and venous volumetric flow rate measurements

\begin{tabular}{lccccc}
\hline Subjects & $\begin{array}{c}\text { TCBF } \\
(\mathbf{m L} / \mathrm{min})\end{array}$ & $\begin{array}{c}\text { R. IJV } \\
(\mathrm{mL} / \mathrm{min})\end{array}$ & $\begin{array}{c}\text { L. IJV } \\
(\mathrm{mL} / \mathrm{min})\end{array}$ & $\begin{array}{c}\text { Total IJV } \\
(\mathrm{mL} / \mathrm{min})\end{array}$ & $\begin{array}{c}\text { Nor IJV } \\
(\%)\end{array}$ \\
\hline $\begin{array}{c}\text { Control } \\
(n=11)\end{array}$ & $789 \pm 116$ & $348 \pm 140$ & $281 \pm 123$ & $628 \pm 72$ & $81 \pm 10$ \\
$\mathrm{IIH}(n=11)$ & $823 \pm 68$ & $333 \pm 150$ & $199 \pm 125$ & $532 \pm 87$ & $65 \pm 7$ \\
$P$ value & 0.414 & 0.817 & 0.14 & 0.011 & 0.001 \\
\hline
\end{tabular}

Note:- L. IJV indicates left internal jugular vein; Nor, normalized; R. IJV, right internal jugular vein; TCBF, total cerebral blood volumetric flow rate. 

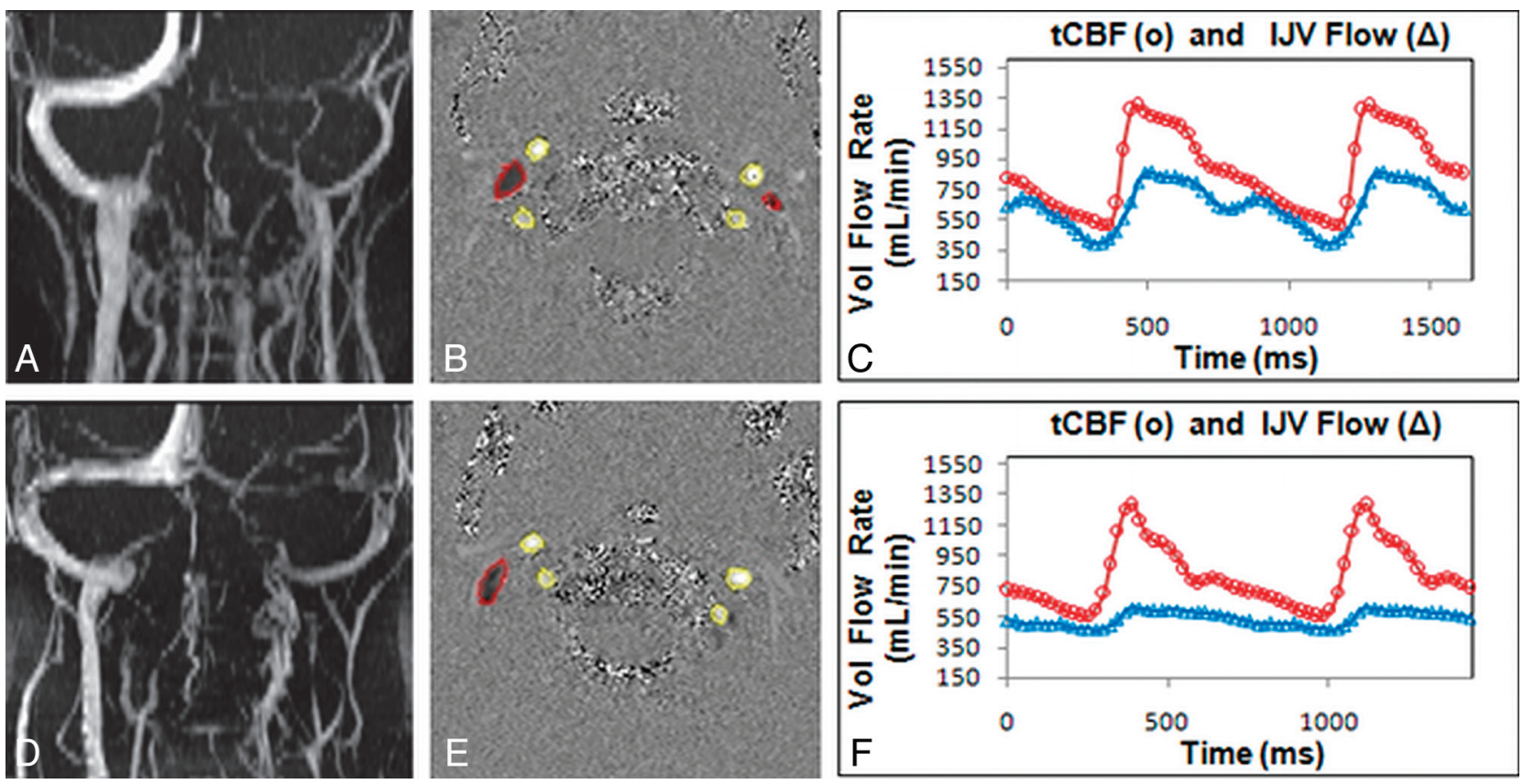

FIG 2. Extracranial MRV, arterial inflow, and venous outflow measurements through the IJVs. An example of MRV $(A, D)$ showing extracranial venous drainage; velocity-encoded phase-contrast images $(B, E)$ showing segmentation of the arterial (bright pixels with yellow outline) and venous (dark pixels with red outline) lumens; and derived volumetric flow rate waveforms (C, F) for total CBF (red) and IJV outflow (blue). Data shown are from a representative control subject (top row) and a patient with IIH (bottom row). Volumetric IJV outflow is lower in the patient with IIH compared with the control subject.

$11 \mathrm{~mL} / \mathrm{min}$, respectively, were not significantly different $(P=.4)$. In both cohorts, venous drainage through the right IJV was larger compared with the left IJV. In contrast to arterial inflow, total venous outflow through the jugular veins was significantly smaller in the patients with IIH compared with the control subjects, $532 \pm 87$ versus $628 \pm 72 \mathrm{~mL} / \mathrm{min}$, respectively, with $P=$ .01 . Normalized jugular cerebral drainage was considerably more significant, $0.65 \pm 0.07$ and $0.81 \pm 0.10, P=.001$, because it accounts for the intersubject variability in total CBF. In 2 patients with IIH and 1 control subject, hypoplasia of 1 transverse sinus was seen in the MRV. However, even with exclusion of these cases, significantly lower jugular drainage in $\mathrm{IIH}$ is maintained (patients with IIH versus controls: $0.64 \pm 0.06$ versus $0.81 \pm 0.10, P=$ $.0009)$.

\section{DISCUSSION}

Disturbed CSF homeostasis, primarily due to impaired CSF absorption, ${ }^{3,4,19}$ is a prevailing hypothesis for the cause of elevated intracranial pressure in IIH. The lack of ventricular expansion, which occurs in other etiologies associated with impaired CSF absorption, but not in IIH, steered the focus away from this hypothesis, despite early data documenting larger subarachnoid spaces in IIH. ${ }^{9}$ The present study employs $3 \mathrm{D}$-volumetric acquisition with 1-mm isotropic resolution, together with a well-tested semiautomated segmentation, to carefully measure intracranial parenchyma and CSF volumes to assess whether extraventricular CSF volume is indeed larger in IIH. Effort was made to study a homogeneous patient population of newly diagnosed untreated overweight female patients with IIH and a well-matched overweight female control group. While ventricular volumes in IIH are maintained at normal range, there is a significant increase in extraventricular CSF and interstitial fluid volumes in the IIH cohort. These results confirm that IIH in obese female subjects is associated with increased intracranial CSF volume that accumulates outside the ventricles. This finding is consistent with the frequent occurrences of posterior globe flattening and papilledema in IIH, because larger subarachnoid CSF spaces adjacent to the floor of the anterior cranial fossa facilitate transmission of the increased CSF pressure to the optic nerve and globe regions. The sensitivity of the extraventricular volume measurement as a marker of IIH is further strengthened when intersubject variability in ICV is accounted for through normalization with respect to ICV. The normalized extraventricular CSF volume had the highest sensitivity and specificity for differentiating between the IIH and the control subjects compared with the other volumetric measures.

The mean CSF volume normalized to the total intracranial volume in the normal cohort of $18 \%$ is larger than previously reported values in healthy female subjects of similar age. ${ }^{10,11}$ Matsumae et $\mathrm{al}^{10}$ found $8 \%$ CSF volume in 6 women (age range $29-40$ years) using 2D imaging (section thickness $3-5 \mathrm{~mm}$ ) and tissue classification based on dual-echo proton attenuation and T2-weighted images. The considerably smaller CSF volume can be attributed to the lower imaging resolution compared with the $1-\mathrm{mm}$ isotropic resolution used in the current study. Moreover, segmentation was based on operator-identified sampling points for each tissue category, thereby potentially biasing the segmentation. In another study, Courchesne et $\mathrm{al}^{11}$ estimated an intracranial CSF volume of $14 \%$ in women of similar age. That study used 3-mm section thickness and a segmentation methodology similar to that used by Matsumae et al, which again may account 
for the lower CSF volume reported by Courchesne et al. ${ }^{11}$ Regardless of differences in normative values of intracranial CSF volume between the present and previous studies, partial volume segmentation bias due to possible differences in cerebral sulci patterns between the cohorts is probably not a reason for the significantly larger extracranial CSF space measured in IIH. On average, the extraventricular CSF space in IIH was larger by $70 \mathrm{~mL}$, or approximately $30 \%$ of the average extraventricular CSF space measured in the control subjects $(220 \mathrm{~mL})$. This difference is approximately 70,000 voxels of $1-\mathrm{mm}$ isotropic resolution, which cannot be attributed to a biased segmentation. Furthermore, the tissue class segmentation method used in the current study accounts for partial volume effects through modeling the intensity distributions of each tissue class to yield a proportion of GM, WM, and CSF in each voxel. ${ }^{16}$

The finding of larger GM volume in IIH is somewhat unexpected. However, GM volume includes interstitial fluid and blood, and therefore larger GM volume may be explained by increased interstitial fluid or blood volume, which has been suggested as a potential mechanism for the elevated intracranial pressure in IIH. ${ }^{3}$ Furthermore, greater water and blood volume content in GM compared with WM potentially explains why GM volume and not WM volume was found to be significantly larger in IIH.

The increased intracranial CSF volume in obesity-related IIH is consistent with a recently reported finding of a smaller spinalcanal/intracranial compliance ratio in obese female patients with IIH compared with control subjects. ${ }^{20}$ Lower spinal canal compliance contribution implies reduced ability to accommodate additional volumes of CSF in the spinal canal, thereby facilitating CSF accumulation in the cranial compartment.

In the current study, total $\mathrm{CBF}$ and relative drainage through the internal jugular veins, the primary drainage channel in the supine position, were assessed to test previously suggested mechanisms of loss of cerebral autoregulation ${ }^{21}$ and/or altered venous hemodynamics in IIH. ${ }^{5}$ A loss of CBF autoregulation was concluded based on a finding of 55\% higher total arterial inflow, with a mean value of $1320 \pm 270 \mathrm{~mL} / \mathrm{min}$, in 12 patients with $\mathrm{IIH}$ categorized to have an extrinsic venous outflow obstruction. ${ }^{21,22}$ In contrast to these studies, a normal mean total arterial inflow of $823 \pm 68 \mathrm{~mL} / \mathrm{min}$ was measured in the current study, suggesting an intact cerebral autoregulation in obesity-associated IIH. A possible explanation for the discrepancy may lie in the different patient cohorts. Bateman's study ${ }^{21}$ included a heterogeneous group of patients of a wide age range (10-52 years) including a 10 -yearold male subject with a total arterial inflow of nearly $2 \mathrm{~L} / \mathrm{min},{ }^{21}$ compared with the homogeneous sample of all-female obese patients with IIH aged 17-44 years in the current study.

While total CBF is normal in the present IIH cohort, drainage through the internal jugular veins is significantly reduced, with a mean value of $0.65 \pm 0.07$ compared with $0.81 \pm 0.1$ in the control cohort. This finding is consistent with an earlier report demonstrating a higher flow through secondary venous outflow channels, such as the epidural and vertebral veins, normalized for total arterial inflow in IIH. ${ }^{5}$ Higher relative drainage through secondary channels compensates for the lower drainage through primary channels, as total venous outflow remains equal to arterial inflow in steady state.

Hypoplasia of 1 transverse sinus observed in $2 \mathrm{IIH}$ subjects and 1 control subject did not influence the finding of reduced jugular drainage in IIH. The strong statistically significant difference $(P<$ $.001)$ between the 2 cohorts is maintained, even with exclusion of the subjects with hypoplasia. This further supports an extracranial cause for the reduced drainage through the jugular veins and increased drainage through secondary veins. Reduced jugular outflow with increased drainage through the smaller secondary veins implies an overall increased resistance to venous outflow. ${ }^{5}$ Higher pleural and central venous pressures, previously reported in obesity-associated IIH, are possible mechanisms for increased venous outflow resistance. ${ }^{23}$

In summary, the current study confirms that IIH in obese women who present with headaches and papilledema is associated with an increased cranial CSF volume that accumulates outside the ventricles. The present study confirms the earlier prediction based on theoretic modeling that intracranial CSF accumulation resulting from impaired CSF homeostasis can occur without ventricular expansion. ${ }^{23}$ In addition to increased cranial CSF volume, the same IIH patient cohort also demonstrated altered venous hemodynamics, suggesting an increased venous outflow resistance. The finding of increased venous outflow resistance, together with the observed increase in GM interstitial fluid volume, supports the hypothesis that interstitial fluid and/or blood volume is increased in IIH. Therefore, the independent observations of increased intracranial CSF volumes and altered venous outflow in the same IIH cohort provide strong evidence that the previously suggested mechanisms of impaired CSF homeostasis and venous outflow coexist in obesity-related IIH.

A limitation of the study is the exclusion of the CSF spaces around the optic nerves and orbits. These regions were not accounted for in the overall CSF volume because they are not identified as part of the cranial vault by the segmentation method. However, these CSF spaces comprise only a small volume and are thus unlikely to change the findings of the study.

\section{CONCLUSIONS}

Quantitative volumetric 3D MR imaging for assessment of intracranial tissues and CSF volumes in conjunction with quantitation of total $\mathrm{CBF}$ and venous drainage through the internal jugular veins demonstrates the coexistence of impaired CSF homeostasis and altered venous hemodynamics in obesity-related IIH. Therefore, these quantitative measures, in addition to previously proposed predictors, ${ }^{24}$ may be useful for establishing a diagnosis of obesity-associated IIH.

\section{ACKNOWLEDGMENTS}

The authors thank Dr. Robert Quencer for departmental support.

\section{REFERENCES}

1. Wall M, George D. Idiopathic intracranial hypertension. Brain 1991;114:155-80

2. Friesner D, Rosenman R, Lobb BM, et al. Idiopathic intracranial hypertension in the USA: the role of obesity in establishing prevalence and healthcare costs. Obes Rev 2010;12:1-8

3. Degnan AJ, Levy LM. Pseudotumor cerebri: brief review of clinical 
syndrome and imaging findings. AJNR Am J Neuroradiol 2011;32:1986-93

4. Pearce JM. From pseudotumour cerebri to idiopathic intracranial hypertension. Pract Neurol 2009;9:353-56

5. Alperin N, Lee SH, Mazda M, et al. Evidence for the importance of extracranial venous flow in patients with idiopathic intracranial hypertension (IIH). Acta Neurochir Suppl 2005;95:129-32

6. Higgins JN, Gillard JH, Owler BK, et al. MR venography in idiopathic intracranial hypertension: unappreciated and misunderstood. J Neurol Neurosurg Psychiatry 2004;75:621-25

7. Karahalios DG, Rekate HL, Khayata MH, et al. Elevated intracranial venous pressure as a universal mechanism in pseudotumor cerebri of varying etiologies. Neurology 1996;46:198-202

8. Walker RW. Idiopathic intracranial hypertension: any light on the mechanism of the raised pressure? J Neurol Neurosurg Psychiatry 2001;71:1-5

9. Silbergleit R, Junck L, Gebarski SS, et al. Idiopathic intracranial hypertension (pseudotumor cerebri): MR imaging. Radiology 1989;170:207-09

10. Matsumae M, Kikinis R, Mórocz IA, et al. Age-related changes in intracranial compartment volumes in normal adults assessed by magnetic resonance imaging. J Neurosurg 1996;84:982-91

11. Courchesne E, Chisum HJ, Townsend J, et al. Normal brain development and aging: quantitative analysis at in vivo $M R$ imaging in healthy volunteers. Radiology 2000;216:672-82

12. Smith JL. Whence pseudotumor cerebri? J Clin Neuroophthalmol 1985;5:55-56

13. Smith SM, Jenkinson M, Woolrich MW, et al. Advances in functional and structural MR image analysis and implementation as FSL. NeuroImage 2004;23:S208-19
14. Woolrich MW, Jbabdi S, Patenaude B, et al. Bayesian analysis of neuroimaging data in FSL. NeuroImage 2009;45:S173-86

15. Smith SM. Fast robust automated brain extraction. Hum Brain Mapp 2002;17:143-55

16. Zhang Y, Brady M, Smith SM. Segmentation of brain MR images through a hidden Markov random field model and the expectation maximization algorithm. IEEE Trans Med Imaging 2001;20:45-57

17. Pieper S, Halle M, Kikinis R. 3D Slicer. In: Proceedings of the 1st IEEE International Symposium of Biomedical Imaging (ISBI): From Nano to Macro. Arlington, Virginia. 2004:632-35

18. Alperin N, Lee SH. PUBS: pulsatility-based segmentation of lumens conducting non-steady flow. Magn Reson Med 2003;49:934-44

19. Levine DN. Ventricular size in pseudotumor cerebri and the theory of impaired CSF absorption. J Neurol Sci 2000;177:85-94

20. Tain RW, Bagci AM, Lam BL, et al. Determination of cranio-spinal canal compliance distribution by MRI: methodology and early application in idiopathic intracranial hypertension. J Magn Reson Imaging 2011;34:1397-404

21. Bateman GA. Association between arterial inflow and venous outflow in idiopathic and secondary intracranial hypertension. J Clin Neurosci 2006; 13:550-56

22. Bateman GA. Arterial inflow and venous outflow in idiopathic intracranial hypertension associated with venous outflow stenoses. J Clin Neurosci 2008;15:402-08

23. Sugerman HJ, DeMaria EJ, Felton III WL, et al. Increased intra-abdominal pressure and cardiac filling pressures in obesity-associated pseudotumor cerebri. Neurology 1997;49:507-11

24. Agid R, Farb RI, Willinsky RA, et al. Idiopathic intracranial hypertension: the validity of cross-sectional neuroimaging signs. Neuroradiology 2006;48:521-27 